\title{
Single-Cell Classification Using Graph Convolutional Networks
}

4 Authors:

5 Tianyu Wang, Computer Science and Engineering Department, University of Connecticut, Storrs, CT,

$6 \quad$ USA, tianyu.wang@uconn.edu

7

8 Jun Bai, Computer Science and Engineering Department, University of Connecticut, Storrs, CT, USA,

9 jun.bai@uconn.edu

10

11 Sheida Nabavi, Computer Science and Engineering Department, University of Connecticut, Storrs, CT,

12 USA, sheida.nabavi@uconn.edu

13

14

\section{Corresponding author:}

16 Sheida Nabavi

17

18

19

20

21

22 


\section{Abstract}

\section{Background}

26 Analyzing single-cell RNA sequencing (scRNAseq) data plays an important role in understanding the

27 intrinsic and extrinsic cellular processes in biological and biomedical research. One significant effort in

28 this area is the identification of cell types. With the availability of a huge amount of single cell

29 sequencing data and discovering more and more cell types, classifying cells into known cell types has

30 become a priority nowadays. Several methods have been introduced to classify cells utilizing gene

31 expression data. However, incorporating biological gene interaction networks has been proved valuable in

32 cell classification procedures.

\section{Results}

34 In this study, we propose a multimodal end-to-end deep learning model, named sigGCN, for cell 35 classification that combines a graph convolutional network $(\mathrm{GCN})$ and a neural network to exploit gene 36 interaction networks. We used standard classification metrics to evaluate the performance of the proposed 37 method on the within-dataset classification and the cross-dataset classification. We compared the 38 performance of the proposed method with those of the existing cell classification tools and traditional 39 machine learning classification methods.

\section{Conclusions}

41 Results indicate that the proposed method outperforms other commonly used methods in terms of

42 classification accuracy and F1 scores. This study shows that the integration of prior knowledge about 43 gene interactions with gene expressions using GCN methodologies can extract effective features 44 improving the performance of cell classification.

\section{Keywords:}

46 Single cell RNA sequencing, cell classification, deep learning, graph convolutional neural network,
47 convolutional
neural
network 


\section{Background}

50 With the explosion of single cell RNA sequencing (scRNAseq) technology in recent years, the

51 unprecedented opportunity for single cell transcription analysis has emerged. The traditional bulk RNA

52 sequencing methods sequence a mix of millions of cells. This results in gene expression of a gene that

53 reflects an average value of the gene expression across all the cells, overlooking the heterogeneity

54 between cells. Different from bulk RNAseq, scRNAseq isolates cells in the first step and performs

55 sequencing on thousands of genes for each cell in the second step. According to different sequencing

56 protocols, hundreds to millions of expression values are gathered for each gene, which allows to identify

57 new cell types [1-3], identify gene regulatory mechanisms, and solve the cellular dynamics of

58 developmental processes.

59 Data analysis for scRNAseq data has attracted much research efforts, such as differential expression

60 analysis, cell clustering, and missing value imputation. In early studies, most researchers have exploited

61 the clustering analysis to categorize cell types [4, 5]. Since most cell sub-types have been identified and

62 features of transcriptomics have become available, the focus on identifying cell subtypes has shifted from

63 detecting new cell types based on clustering-based methods to discovering cell-specific expression

64 signatures based on classification-based methods. Classification is a supervised learning method that

65 requires plenty of data to perform the training process. Compared to clustering, a well-trained classifier

66 based on public annotated datasets can efficiently and accurately identify the unlabeled cells, even the

67 unlabeled data are from different platforms and samples [6].

68 Few cell classification methods have been proposed for scRNAseq data that can be grouped into two

69 categories. One group of cell classification methods, including scPred [7], scID [8] and CasTLe [9] are

70 based on traditional supervised machine learning methods, such as random forest (RF), support vector

71 machine (SVM) and k nearest neighbors (KNN) [7, 10]. scPred [7] combines a feature selection and SVM

72 with radial kernel to classify cell types. scID [8] applies the fisher linear discriminant analysis for cell

73 classification. CasTLe [9] combines the data preprocessing and classifies cells based on the XGBoost [11]

74 classifier. The other group of methods are based on cluster level similarity measurements, for example 
75 SingleR [12] and scmap [13]. SingleR [12] employs Spearman correlation to calculate the similarity

76 between cells. There are two methods in the scmap [13] package, scmapcluster and scmapcell.

77 scmapcluster, first, constructs a virtual cell for each cell type based on the median expression values of

78 the genes across that specific cell type. Then, it computes the cell similarity between the cells that need to

79 be labeled and the virtual cells. The labels are assigned by the highest similarity. Scmapcell computes the

80 cell similarity directly and employs the KNN method to classify the cells. Also, some studies have used

81 marker genes as prior knowledge for cell classification [14, 15]. However, the marker genes are not

82 always available. These classification methods are not scalable and they work well only when dealing

83 with small datasets with the prerequisite of reasonable feature selection.

84 With the huge progress of deep learning in computer vision and image classification, a variety of deep

85 learning models have been explored and designed. A deep learning network learns high-level features

86 from data and thus does not need the domain knowledge to select the features, which is beneficial for the

87 classification of a huge number of samples. A recent cell classification method, ACTINN [16], employs a

88 fully connected neural network for cell type classification.

89 Even though gene expression values are regulated by gene networks, classification based on gene

90 expression values ignores this prior gene interaction knowledge. To combine the prior knowledge of

91 biological networks and gene expression, we introduce the use of graph convolutional network (GCN) [17,

92 18]. The gene network and gene expression can be considered as different views of the data. To learn

93 useful features to represent complex data, multiple views of data need to be considered. This has

94 motivated us to develop a multimodal deep learning model based on GCN and neural network (NN)

95 methodologies. A GCN is an extension of convolution on the graph domain. The GCN approaches have

96 been applied to address biological problems such as predicting protein functions [19, 20].

97 For single cell classification, this paper proposes a new GCN-based end-to-end multimodal deep

98 learning model. The proposed model learns the feature map based on both the gene expression values and

99 the gene-gene interaction structure. The proposed multimodal GCN model employs a GCN paralleled

100 with an NN model. Due to the localization property of learning filters in the GCN, it can extract local 
101 features based on the prior knowledge - the genomics interaction network. Hence, employing only a GCN

102 model fails to capture global features. The quality of the extracted features depends on not only the GCN

103 filters but also the completeness of the underlying genomics interaction network. The features extracted

104 by a fully connected NN can represent the global connections but neglect the inner interactions. As a

105 result, to have a more precise feature embedding, we utilize a parallel structure that consists of a GCN and

106 an NN. In other words, combining localized features extracted from a GCN and global features extracted

107 from an NN for classification conquers the limitations of GCN and NN models. We evaluate the

108 performance of the proposed method using seven single cell datasets. We compare the performance of the

109 proposed method with those of seven scRNAseq data classification tools and four conventional

110 classification methods.

\section{Methods}

\section{Network structure}

114 The overall structure of the proposed model is shown in Figure 1. The proposed classification model

115 consists of two parallel networks: a GCN and an NN. Gene expression values and the gene adjacency

116 network are the inputs of the GCN; while the gene expression values are the inputs of the NN. The

117 features learned by the GCN and the NN are concatenated and then connected with a fully connected

118 layer. The output layer outputs the predicted cell type of an input cell.

\section{Gene adjacency matrix}

121 We utilized the STRING database [21] to build the weighted gene adjacency matrix. The size of the

122 adjacency matrix is $N \times N$, where $N$ is the number of the genes. The elements in the matrix represent the

123 confident score between pairs of genes extracted from the gene-gene interaction database. Then, we

124 normalized the weights in the adjacency matrix by row sums. Using the normalized adjacency matrix, we

125 built a weighted graph that the nodes are genes (proteins), the edges represent the connection between

126 genes and the normalized confidence scores are weights of edges. We regarded the genes that have no 
127 neighbors in the matrix as the singletons in the network. We need to mention that since we chose top $N$

128 genes with highest variances in expression values for training (explained in the Data preprocessing

129 section), there are singletons in the graph. We discussed the model performance including the singletons

130 and excluding the singletons in the results section.

131 To explore the benefit of using the gene network, we also constructed the gene adjacency matrix using

132 the gene co-expression similarity, where the elements in the gene adjacency matrix are the gene cosine

133 similarities. To compute the cosine similarity between two genes, we considered the gene expressions

134 across all the cells as vectors. Then the cosine similarity of a pair of genes were computed by $(\mathbf{x}$.

$135 \mathbf{y}) /(\|\mathbf{x}\|\|\mathbf{y}\|)$, where $\mathbf{x}$ and $\mathbf{y}$ are the expression vectors of the two genes. We filtered out edges for pairs

136 of genes whose cosine similarities are less than 0.6. We compared the model performance when using the

137 gene interaction network with that of when using the gene co-expressions to construct the gene graph.

\section{Graph convolutional network}

140 In this work, to extract the features of expression values incorporating the gene interaction network, we

141 developed a GCN-based autoencoder model. For the GCN analysis, gene expression values are assigned

142 as the features of the nodes. The encoder part of the GCN model consists of a GCN layer followed by a

143 maxpooling layer, a flatten layer and a fully connected (FC) layer. While the decoder part consists of a FC

144 layer to reconstruct the gene expression values.

145 The GCN layer consists of graph convolution and pooling operations. The gene expression matrix can

146 be represented as $\mathbf{X} \in R^{N \times M}$, where $N$ is the number of genes and $M$ is the number of cells. Considering

147 graph $G=(V, E)$, where $V$ represent the vertices (genes) and $E$ represents the edges between the

148 vertices, the gene expression values can be regarded as the vertex features. The adjacency matrix $\mathbf{A} \in$

$149 R^{N \times N}$ is used to represent the edges, i.e. the connections between the genes, constructed from the gene-

150 gene interaction network. The Laplacian matrix is defined as $\mathrm{L}=\mathrm{D}-\mathrm{A}$, where $\mathrm{D} \in R^{N \times N}$ is a diagonal

151 matrix. In the diagonal matrix $\mathrm{D}$, the values on the diagonal represent the number of edges that connect to 
152 a vertex. The Laplacian matrix is further normalized to $\mathbf{L}=\mathbf{I}+\mathbf{D}^{-1 / 2} \mathbf{A D}^{1 / 2}$, where $\mathbf{I} \in R^{N \times N}$ is the

153 identity matrix. Because the normalized Laplacian matrix is a real symmetric positive semidefinite matrix,

154 it can be factorized to:

$$
\mathbf{L}=\mathbf{U} \boldsymbol{\Lambda} \mathbf{U}^{T}
$$

155 where $\mathbf{U}=\left(\mathbf{u}_{1}, \ldots \mathbf{u}_{l}, \ldots \mathbf{u}_{n}\right)$ contains a set of orthonormal eigenvectors, $\mathbf{U} \mathbf{U}^{T}=\mathbf{I}$, and $\boldsymbol{\Lambda}$ is the 156 eigenvalue matrix $\Lambda=\operatorname{diag}\left(\lambda_{1}, \ldots \lambda_{l}, \ldots \lambda_{n}\right)$.

157 The graph convolutional theory comes from the spectral convolution theory. Like the convolution of 158 two one-dimensional signals, given the Fourier transformation of a graph as $\widehat{\mathbf{x}}=\mathbf{U}^{T} \mathbf{x}$ [22] and the 159 inverse Fourier transformation $\mathbf{x}=\mathbf{U} \hat{\mathbf{x}}$, the convolution on the graph is defined as [18]:

$$
\mathbf{x} * \mathbf{h}=\mathbf{U}\left(\mathbf{U}^{T} \mathbf{h} \odot \mathbf{U}^{T} \mathbf{x}\right)=\mathbf{U} h(\boldsymbol{\Lambda}) \mathbf{U}^{\mathrm{T}} \mathbf{X}, \#(2)
$$

160 where $\odot$ is the Hadamard (element-wise) product, and $\mathbf{x}$ represents the $N$-dimensional vector on the 161 graph, i.e. the gene expression values of a cell in this work. $h(\Lambda)$ is a diagonal matrix that is denoted as 162 the convolution kernel of the transformation. In general, the designation of the convolution kernel decides 163 the computation cost of the graph convolution. The convolution kernel can be designed as $h(\Lambda)=$ $164 \sum_{k=0}^{K-1} \beta_{k} \Lambda^{k}$ [18], where $K$ is the receptive field of the convolution kernel (i.e. the order of the neighbors 165 that are computed) and $\beta_{k}$ s are the polynomial coefficients. The kernel is approximated by the Chebyshev 166 polynomial as following to further decrease the computation cost:

$$
h(\boldsymbol{\Lambda})=\sum_{k=0}^{K-1} \beta_{k} T_{k}(\widetilde{\Lambda}), \#(3)
$$

167 where $T_{k}(\widetilde{\Lambda})$ is the $k^{\text {th }}$ order of the Chebyshev polynomial which is recursively defined as $T_{k}(\widetilde{\Lambda})=$ $1682 \widetilde{\Lambda} T_{k-1}(\widetilde{\Lambda})-T_{k-2}(\widetilde{\Lambda}), T_{0}(\widetilde{\Lambda})=\mathbf{I}, T_{1}(\widetilde{\Lambda})=\widetilde{\Lambda}[23]$. The $\widetilde{\Lambda}$ is the rescaled diagonal eigenvalue matrix 169 of the Laplacian matrix, which is defined as $\widetilde{\Lambda}=2 \Lambda / \lambda_{\max }-\mathbf{I}$. The parameters, $\beta_{k} \mathrm{~s}$, are learned during 170 the training process. Therefore, when substituting Equation (3) into Equation (2), the convolution on the 171 graph is computed by: 


$$
\mathbf{x} * \mathbf{h}=\mathbf{U} \sum_{k=1}^{K-1} \beta_{k} T_{k}(\widetilde{\Lambda}) \mathbf{U}^{T} \mathbf{x}=\sum_{k=0}^{K-1} \beta_{k} T_{k}(\tilde{\mathbf{L}}) \mathbf{x}, \#(4)
$$

172 where $\tilde{\mathbf{L}}=2 \mathbf{L} / \lambda_{\max }-\mathbf{I}, T_{k}(\tilde{\mathbf{L}})=2 \tilde{\mathbf{L}} T_{k-1}(\tilde{\mathbf{L}})-T_{k-2}(\tilde{\mathbf{L}}), T_{0}(\tilde{\mathbf{L}})=\mathbf{I}, T_{1}(\tilde{\mathbf{L}})=\tilde{\mathbf{L}}$. With this substitution,

173 the multiplication of the matrices is no longer needed, and the convolution computation depends on the

$174 K^{\text {th }}$ order neighbors of the vertices. In this way, the convolution is transformed to the weighted summation

175 of the $K$-hop neighbors which can keep the spatial localization. Then, the output of the graph convolution

176 layer can be written as:

$$
\mathbf{Z}^{\prime}=\sigma\left(\sum_{k=0}^{K-1} \beta_{k} T_{k}(\tilde{\mathbf{L}}) \mathbf{x}\right)
$$

177 where $\sigma(\cdot)$ is the activation function, and $\mathbf{x}$ is the input of the graph, i.e. the gene expression values of a

178 cell. The receptive field of the kernel, $K$, can be considered as a hyper-parameter which is chosen as 5 in

179 this study. In this work, we employed multiple convolution kernels $(F=5)$, then the dimension of the

180 graph convolutional layer output feature map is $N \times F$. We also used a maxpooling layer with size

$181 p=8$ after the graph convolutional layer, which means we clustered $p$ nodes (genes) in the graph into

182 one big node. Therefore, the feature map $(\mathbf{Z})$ generated from the proposed GCN has the dimension of the

183 number of nodes after pooling $(N / p)$ by the number of features $(F=5), \mathbf{Z} \in R^{N / p \times F}$.

184 Since the main goal is classification on the graph level (cell level) but not on the node level (genes), 185 this feature map is flattened and then connected with a dense layer with the size of 32 neurons to reduce 186 the dimension. Therefore, the final feature, $\theta_{1}$ (shown in Figure 1), outputted by the GCN is a vector with 187 the size of 32.

\section{Reconstruction of gene expression}

190 In the GCN-based autoencoder, the decoder part is utilized to reconstruct the node attributes (gene 191 expressions). The features learned by the GCN (encoder part), i.e. the hidden layer shown in Figure 1, is 192 fully connected to an output layer to build the decoder network. The output of the decoder has the same 
193 dimension as the input, which is the number of input genes, $N$. We utilized the mean squared error (MSE)

194 as the loss function for this task:

$$
L_{r e c}=\sum_{i}\left(\mathbf{x}_{i}-\hat{\mathbf{x}}_{i}\right)^{2}, \#(6)
$$

195 where $\mathbf{x}_{\boldsymbol{i}}$ is the vector of gene expression values of cell $i$ and $\hat{\mathbf{x}}_{i}$ is the vector of reconstructed gene

196 expression values.

198 Neural network

199 The inputs of the NN (Figure 1) are the gene expression values of the top $N$ (default is 1000) variant

200 genes. Since the number of cells varies in different datasets and not all datasets have a large number of 201 cells, we designed a shallow NN to avoid the overfitting problem. The network has two fully connected 202 layers with the size of 256 and 32 neurons, respectively. The output of each layer is activated by the 203 ReLU function. Therefore, the final feature from the NN, $\theta_{2}$ (shown in Figure 1), is of size 32.

\section{Fully connected integrative layer for classification}

206 The features learned by the NN are concatenated with the features learned by the GCN in the integration

207 layer $\theta_{3}$ (shown in Figure 1). The concatenated features are the input to the final output classification layer 208 with the size of the number of the classes. Assume that there are $n$ classes of cell types, the output layer is 209 of size $n$ and the corresponding probability array, $\mathbf{p}=\left[p_{1} \ldots p_{n}\right]$, is calculated over the $n$ output neurons 210 using the softmax activation function. The predicted label of cell $i$ is the class that has the highest 211 probability in array $\mathbf{p}$, which is $\hat{y}_{i}=\operatorname{argmax}(\mathbf{p})$, shown in Figure 1. For its corresponding true class $y_{i}$,

212 the loss is computed by the negative log-likelihood function defined as:

$$
L_{c l a}=-\log \left(p_{y_{i}}\right) \text {,\#(7) }
$$

213 where $p_{y_{i}}$ is the output probability of class (cell type) $y_{i}$.

\section{Loss functions and model training}


216 We also used the regularization loss $L_{\text {reg }}$ to regularize the parameters and prevent overfitting. Assume W

217 consists of all the parameters in the model - including the parameters in the GCN, the NN, the decoder,

218 and the integration layer - and $w_{j}$ represents each of the parameters in the model, the regularization loss

219 is defined as:

$$
L_{r e g}=\sum_{j} w_{j}{ }^{2} . \#(8)
$$

220 The total loss is a combination of the loss of classification, the loss of the reconstruction, and the loss of

221 regularization:

$$
L=\lambda_{1} L_{c l a}+\lambda_{2} L_{r e c}+\lambda_{3} L_{r e g}, \#(9)
$$

222 where $\lambda_{1}, \lambda_{2}$ and $\lambda_{3}$ are the weights for each loss function. In this study, $\lambda_{1}$ and $\lambda_{2}$ are set to 1 , and $\lambda_{3}$ is

223 set to 0.0001 . The loss is computed and backpropagated to update the parameters. We used the SGD

224 optimizer [24] to train the end-to-end model. The mini-batch size and epoch were chosen as 64 and 50,

225 respectively. To train the model, we split the datasets into $80 \%$ as the training dataset, $10 \%$ as the

226 validation dataset, and $10 \%$ as the testing dataset. We first used training dataset to train the model, and

227 then tested the model on the validation dataset. We tuned the hyperparameters based on the performance

228 of the model on the validation dataset. After tuning, we fixed the hyperparameters and evaluated the

229 performance of the model on the testing dataset.

\section{Datasets}

232 In this section, we describe the datasets and also the data preprocessing used in this work.

\section{Single cell datasets}

234 To evaluate the performance of the proposed classification method, we used seven datasets which are

235 from different sequencing protocols, across different species: human and mouse. All the datasets can be

236 downloaded from https://doi.org/10.5281/zenodo.3357167 [25]. The datasets are across different cell

237 populations and have a different number of cells and genes. Two of them are large scale datasets

238 (Zheng68K and Zhengsorted) containing several ten-thousands of cells, and only one of the dataset 
239 (Zhengsorted) has validated ground truth while others use labels obtained by employing a clustering

240 method on some marker genes.

242 Pancreatic datasets.

243 We used five scRNAseq datasets from the human pancreas and mouse pancreas. The datasets are from

244 different individual samples using different sequencing protocols. The BaronMouse [26] and the

245 BaronHuman [26] datasets are from the mouse and human pancreas, respectively. The cells are sequenced

246 using the inDrop protocol. The filtered BaronMouse dataset has expression data of 1,886 cells and 14,861

247 genes from 13 cell populations. In the BaronHuman dataset, there are expression data of 8,569 cells and

248 17,499 genes from 14 cell populations after filtering. The Muraro [27], Segerstolpe [3], and Xin [28]

249 datasets are all from the human pancreas. The Muraro dataset is available on GEO with access number

250 GSE85241 and sequenced by the CEL-Seq2 protocol. After filtering the dataset, there are expression data

251 of 2,122 cells and 18,915 genes, where the cells are annotated to 9 classes. The Segerstolpe dataset

252 includes expression data of 2,133 cells and 22,757 genes, sequenced by the SMART-Seq2 protocol, from

25313 cell populations. The last human pancreas dataset is the Xin dataset which is sequenced by the

254 SMARTer protocol. There are 4 types of 1,449 cells in the dataset. For these datasets, we used the cell

255 labels given by the authors as ground truth. Note that the marker genes and computational methods are

256 used to assign these labels.

258 Peripheral blood mononuclear cell.

259 Human peripheral blood mononuclear cells contain heterogeneous cell populations and play an important

260 role in investigating immunology and infectious disease. We used two datasets [29], Zhengsorted and

261 Zheng68K, which were sequenced by the 10x Chromium protocol [30]. In the Zhengsorted dataset, the

262 authors extracted 10 cell populations using antibody based bead enrichment and validated the ten cell

263 types using the FACS sorting. The purified 10 populations were used to generate a large set of single cells

264 individually. Similar to the work in [25], each cell population has 2,000 cells and were mixed together to 
265 have an even combined dataset, therefore, there are 20,000 cells of 10 cell classes. The Zheng68K dataset

266 is a large-scale scRNAseq dataset that includes expression of 65,943 cells and 20,387 genes. The cells

267 were labeled by [29] as 11 cell populations. We used the given annotations as ground truth.

Data preprocessing

270 All the datasets were filtered by cells and genes. First, we removed the unlabeled cells, and the cells that

271 were labelled as debris and doublets. Also, we removed the genes that have zero expression values across

272 all the cells. Then, we transformed the gene expression values into the log scale and normalized each

273 dataset by min-max scaling. After calculating the variances of the genes across all the cells, we sorted the

274 variances in descending order and chose the top 1,000 genes as the input of the classifiers. For the graph-

275 based method, we constructed the gene adjacency network from the selected genes.

277 Complexity of the Datasets

278 We used the density plot to describe the complexity of the datasets. We calculated the mean expression

279 value of each gene across the cells from the same cell population, as the centroid. Then, we generated a

280 matrix with the dimension of the number of genes by the number of the cell populations for each dataset.

281 The distances between the cell populations were computed using this matrix. Figure 2 shows the density

282 of distance between the centroids of the cell populations. The high peaks of the datasets show that the cell

283 populations are similar to each other and are not easy to separate. We can observe that the Zhengsorted

284 and Zheng68K datasets are the most complex datasets.

286 Results and discussion

287 For performance comparison, we used seven tools developed for scRNAseq data classification: scPred [7], 288 scID [8], CaSTLe [9], singleR [12], scmapcluster [13], scmapcell [13], and ACTINN [16]. We also used 289 conventional supervised classifiers including RF, linear SVM (SVM-linear), radial basis function kernel- 
291 implement the traditional classifiers.

292 To investigate the generalizability of the methods, we used the within-dataset classification and cross293 dataset classification to evaluate the performance of the methods. In our within-dataset classification, we

294 randomly split each dataset for training and testing the models. We used the same training and testing 295 datasets for all the methods. In our cross-dataset classification study, first, we combined the four human 296 pancreas datasets (Xin, BaronHuman, Muraro, and Segerstolpe) and then, used three of them as the 297 training dataset and the remaining one as the testing dataset.

\section{Metrics to evaluate the performance of classification}

300 We used accuracy, F1 score, precision, and recall metrics to evaluate the performance of the classifiers. 301 They are defined as: precision $=\mathrm{TP} /(\mathrm{TP}+\mathrm{FP})$, recall $=\mathrm{TP} /(\mathrm{TP}+\mathrm{FN})$, accuracy $=$ $302(\mathrm{TP}+\mathrm{TN}) /(\mathrm{TP}+\mathrm{TN}+\mathrm{FP}+\mathrm{FN}), \mathrm{F} 1=(2 \times$ precision $\times$ recall $) /($ precision + recall $)$, where $\mathrm{TP}, \mathrm{FP}, \mathrm{TN}$ and

303 FN denote true positive, false positive, true negative, and false negative, respectively. We used the 304 accuracy and the median of F1 scores across all the classes to evaluate the overall performance of a 305 classifier. We also showed the median precisions and the median recalls in the results section.

307 We evaluated the performance of the classification within each dataset individually. Figure 3 shows the 308 performance of the scRNAseq data classifier tools and the conventional classifiers on the Zhengsorted 309 dataset in comparison to our proposed classifier, sigGCN, in terms of accuracy, F1, precision, and recall.

310 Note that the Zhengsorted dataset is the only dataset with validated ground truth cell types. For other 311 datasets, the labels are obtained by employing a clustering method on the marker genes. The accuracy 312 (0.922), median F1 (0.965), precision (0.970), and recall (0.972) of our model are improved compared to

313 not only the traditional machine learning methods but also the methods that are developed specifically for 314 cell classification. Our model performs better than the fully connected neural network based method, i.e.

315 ACTINN (accuracy $=0.845, \mathrm{~F} 1$ score $=0.892$, precision $=0.913$, recall $=0.886$ ). 

perform well using the Zheng68K dataset because it is the most complex dataset as shown in Figure 2. reason is that the conventional methods are not scalable for large datasets. The Zhengsorted dataset with 20,000 cells is a large scale scRNAseq dataset which benefits the deep learning based methods. most of the datasets.

Almost all the methods perform well on the Xin, Segerstolpe, and Muraro datasets and there are not

331 population in these datasets. As can be seen in Figure 2 the distances between clusters are higher in these

332 datasets compared to the rest. Interestingly, for more complex datasets, Zhengsorted, Zheng68K, in which

333 the Euclidian distances between clusters are lower, our proposed method performs significantly better as

334 can be seen in Table 1.

335 Table 1. Accuracy of the eight scRNAseq data classifier tools and the four conventional classifiers

336 on the seven datasets $(N=\mathbf{1 0 0 0})$

\begin{tabular}{|l|l|l|l|l|l|l|l|}
\hline Methods & Zhengsorted & Zheng68K & BaronHuman & Muraro & Segerstolpe & BaronMouse & Xin \\
\hline sigGCN & $\mathbf{0 . 9 2 2}$ & $\mathbf{0 . 7 5 2}$ & $\mathbf{0 . 9 7 9}$ & $\mathbf{0 . 9 9 1}$ & $\mathbf{0 . 9 7 7}$ & 0.974 & 0.993 \\
\hline scID & 0.721 & 0.484 & 0.46 & 0.577 & 0.285 & 0.286 & 0.986 \\
\hline scPred & 0.515 & 0.140 & 0.86 & 0.915 & 0.827 & 0.862 & 0.91 \\
\hline CasTLe & 0.836 & 0.736 & 0.971 & 0.972 & 0.953 & 0.91 & 0.993 \\
\hline SingleR & 0.723 & 0.388 & 0.951 & 0.977 & 0.953 & 0.868 & $\mathbf{1}$ \\
\hline scmapcluster & 0.395 & 0.409 & 0.946 & 0.962 & 0.949 & 0.905 & 0.931 \\
\hline scmapcell & 0.727 & 0.246 & 0.895 & 0.972 & 0.949 & 0.778 & 0.952 \\
\hline
\end{tabular}




\begin{tabular}{|l|l|l|l|l|l|l|l|}
\hline ACTINN & 0.845 & 0.737 & 0.977 & 0.991 & 0.958 & $\mathbf{0 . 9 8 4}$ & $\mathbf{1}$ \\
\hline RF & 0.835 & 0.69 & 0.968 & 0.981 & 0.967 & 0.968 & 0.993 \\
\hline SVM-linear & 0.859 & 0.652 & 0.824 & 0.981 & 0.383 & 0.704 & $\mathbf{1}$ \\
\hline SVM-rbf & 0.884 & 0.677 & 0.93 & 0.981 & 0.841 & 0.794 & $\mathbf{1}$ \\
\hline KNN & 0.824 & 0.594 & 0.953 & 0.991 & 0.935 & 0.926 & $\mathbf{1}$ \\
\hline
\end{tabular}

Table 2. Median F1 of the eight scRNAseq data classifier tools and the four conventional classifiers

on the seven datasets $(N=1000)$

\begin{tabular}{|l|l|l|l|l|l|l|l|}
\hline Methods & Zhengsorted & Zheng68K & BaronHuman & Muraro & Segerstolpe & BaronMouse & Xin \\
\hline sigGCN & $\mathbf{0 . 9 6 5}$ & $\mathbf{0 . 7 7 6}$ & 0.977 & $\mathbf{1}$ & $\mathbf{1}$ & 0.969 & 0.995 \\
\hline scID & 0.66 & 0.535 & 0.22 & 0.578 & 0 & 0 & $\mathbf{1}$ \\
\hline scPred & 0.568 & 0.105 & 0.833 & 0.932 & 0.8 & $\mathbf{0 . 9 7}$ & 0.784 \\
\hline CasTLe & 0.834 & 0.667 & 0.956 & 0.967 & 0.965 & 0.848 & 0.997 \\
\hline SingleR & 0.678 & 0.335 & 0.946 & 0.984 & $\mathbf{1}$ & 0.898 & $\mathbf{1}$ \\
\hline scmapcluster & 0.729 & 0.357 & 0.9 & 0.997 & 0.965 & 0.88 & 0.991 \\
\hline scmapcell & 0.305 & 0.198 & 0.95 & 0.993 & 0.977 & 0.942 & 0.708 \\
\hline ACTINN & 0.892 & 0.753 & $\mathbf{1}$ & 0.97 & $\mathbf{1}$ & $\mathbf{1}$ & 0.997 \\
\hline RF & 0.853 & 0.646 & 0.956 & 0.987 & 0.993 & 0.984 & 0.997 \\
\hline SVM-linear & 0.868 & 0.663 & 0.362 & $\mathbf{1}$ & 0.059 & 0.238 & $\mathbf{1}$ \\
\hline SVM-rbf & 0.9 & 0.671 & 0.906 & $\mathbf{1}$ & 0.772 & 0.695 & $\mathbf{1}$ \\
\hline KNN & 0.795 & 0.613 & 0.928 & $\mathbf{1}$ & 0.961 & 0.889 & $\mathbf{1}$ \\
\hline
\end{tabular}

Using the BaronHuman dataset, our model shows the best performance against other methods with an accuracy of 0.979 and median F1 of 0.977 . The RF and KNN have good accuracy but could not hold the high median precision and median recall values. This is because the accuracy is calculated based on the global TPs and TNs, but the medians of all the paired scores indicate that some classes with a small number of members were not classified well using the conventional methods. Our model reduces FN predictions and increases TP predictions, which notably improves the median recall. In addition, the FPs of our model are also reduced that substantially enhances the precision. Therefore, the median F1, median precision and recall are higher compared to those of the other methods using the BaronHuman dataset.

The performance of our model using the BaronMouse dataset is comparable to that of the ACTINN and better than those of the other methods. Overall, our proposed model shows the best or near the best performance using all the datasets, especially for complex datasets that have large cell numbers and more classes. The performance of sigGCN is improved significantly in comparison to the pure neural network which indicates that integrating the prior knowledge of gene interactions can learn a better representation of data. The feature representation learned by convolution through the gene interaction structure is not 
355 redundant with that learned through the expression values. The parallel structure enriches extracting

356 effective features during the training.

357 We also evaluated the performance of the proposed method when constructing the gene graph using i)

358 the gene cosine similarity, and ii) the gene interaction network without singleton genes. Results show that

359 employing the gene interaction network (including singleton genes) provides the best performance (as can

360 be seen from Additional file 1: Tables S1 - S4). To evaluate the performance of the proposed parallel

361 network compared to employing only a GCN, we compared the performance of sigGCN with that of a

362 pure-GCN. Results in Additional file 1: Table S5 show that the parallel network outperforms the pure

363 GCN model, which indicates that the parallel network (NN and GCN) can capture global and local

364 features.

365 We need to mention that scPred, scID, scmapcluster, and scmapcell provide the function of rejection

366 which means they predict a cell class as "unassigned". We computed the accuracy and F1 scores based on

367 the results of including these unassigned cells in our comparison (Table 1 and Table2). Since our method

368 outputs the probability of cell class assignments, we also provide an additional function to predict a cell

369 class as "unassigned" by setting a threshold of prediction. We set the threshold as 0.65 which means if a

370 cell does not have a probability of prediction larger than 0.65 , then the cell will be predicted as

371 "unassigned". We computed accuracy and F1 scores when not including the unassigned cells for these

372 four methods and our method with the "unassigned" function (shown in Additional file 2 Table). Results

373 show that our method has the smallest unassigned rate and the best or near the best accuracy and F1.

\section{Confusion matrix and ROC analysis}

376 Since the Zhengsorted dataset is the one that has the ground truth, we provided more details on the

377 performance of the methods using this dataset in this section. The confusion matrix of the ten classes

378 using our proposed method, sigGCN, is shown in Figure 4. The confusion matrices of the other

379 scRNAseq classifiers are shown in Additional file 1: Figures S7-S8, and those of the conventional

380 methods are shown in Additional file 1: Figure S9. As can be seen in Figure 4, using our model the TP 
rate of class 0 , to class 9 are $99.5 \%, 100 \%, 99.5 \%, 69.3 \%, 80.9 \%, 84.6 \%, 97.1 \%, 99.0 \%, 97.4 \%, 92.0 \%$, respectively. Class 3, class 4, and class 5 have confusion with each other because CD4+ cells have high heterogeneity.

To further investigate the performance of the methods, we executed the ROC analysis for each class using the Zhengsorted dataset. Figure 5(a) shows the ROCs for each class using our proposed method.

We also compared our model with the other methods in terms of AUC and the ROC analysis of each class.

387 The ROC analysis for class 4 (CD4+/CD25 T Reg) using all the methods is shown in Figure 5(b). We

388 only showed the performance of six methods (two scRNAseq classifiers and four conventional classifiers)

389 which provides the output classification probability. Our model shows the best AUC of 0.9872 in the

390 ROC analysis of class 4. To test the significance of the difference between the AUCs under the curves,

391 we used the McNeil \& Hanley's test [32] and online service on http://vassarstats.net/roc_comp.html. The

392 p-values of the tests between our method sigGCN and each method are shown in Figure 5(b). The ROC

393 analysis of class 3, class 5, class 6, and class 8 are also shown in Additional file 1: Figure S10.

\section{Performance of cross-dataset classification}

396 In order to examine the generalization of the proposed model, we evaluated the performance of cross-

397 dataset classification, which is a more realistic scenario. Since the Xin, BaronHuman, Muraro, and

398 Segerstolpe datasets are all from the human pancreas, we used these four datasets for the validation. The

399 common cell types among these four datasets are alpha, beta, delta, and gamma, so we extracted the four

400 cell types from each dataset for combination. Before combining the datasets, we preprocessed the data

401 using the log-transformation and normalized each dataset by min-max scaling to make the four datasets in

402 the same level. We run four experiments, and in each experiment, we used three of the four datasets as the

403 training dataset and the remaining one as the testing dataset. Additional file 1: Figures S11-S14 show the

404 performance of the methods in terms of accuracy, median F1, median precision, and median recall score.

405 The results of the accuracy are shown in Table 3 and the median F1 scores are shown in Table 4,

406 respectively. We included the unassigned cells when computing the accuracy and F1 scores for the scPred, 
407 scID, scmapcluster, and scmapcell. We also provided the results of not including the unassigned cells in

408 Additional file 3.

409 All the methods except the scmapcluster, scmapcell, and KNN perform nearly perfect in terms of

410 accuracy when training on the BaronHuman, Muraro, and Segerstolpe and testing on the Xin dataset.

411 Providing a near $100 \%$ accuracy, F1, precision, and recall, our model shows the best performance

412 compared to the other methods. Our model ranks the third and the second in terms of accuracy when

413 testing on the Muraro and Segerstolpe datasets, respectively, with a slight difference of 0.003 . Overall,

414 our model achieves the best or near the best performance in terms of accuracy and median F1 in the cross-

415 dataset classification evaluation.

Table 3. Accuracy of the eight scRNAseq data classifier and the four conventional classifiers tools

\section{8 on the four experiments $(N=1000)$}

\begin{tabular}{|l|l|l|l|l|}
\hline Training Dataset & $\begin{array}{l}\text { BaronHuman+ } \\
\text { Muraro+ Segerstolpe }\end{array}$ & $\begin{array}{l}\text { Xin+ Muraro+ } \\
\text { Segerstolpe }\end{array}$ & $\begin{array}{l}\text { Xin+ BaronHuman+ } \\
\text { Segerstolpe }\end{array}$ & $\begin{array}{l}\text { Xin+ } \\
\text { BaronHuman+ } \\
\text { Muraro }\end{array}$ \\
\hline Testing Dataset & Xin & BaronHuman & Muraro & Segerstolpe \\
\hline sigGCN & $\mathbf{0 . 9 9 7}$ & $\mathbf{0 . 9 8 7}$ & 0.974 & 0.993 \\
\hline scID & 0.989 & 0.747 & 0.97 & 0.979 \\
\hline scPred & 0.945 & 0.467 & 0.92 & 0.814 \\
\hline CasTLe & 0.99 & 0.944 & $\mathbf{0 . 9 7 7}$ & 0.992 \\
\hline SingleR & 0.995 & 0.984 & $\mathbf{0 . 9 7 7}$ & $\mathbf{0 . 9 9 6}$ \\
\hline scmapcluster & 0.196 & 0.003 & 0.051 & 0.568 \\
\hline scmapcell & 0.756 & 0.421 & 0.64 & 0.367 \\
\hline ACTINN & 0.993 & 0.984 & 0.974 & 0.992 \\
\hline RF & 0.982 & 0.941 & 0.947 & 0.938 \\
\hline SVM-linear & 0.994 & 0.979 & 0.972 & 0.992 \\
\hline SVM-rbf & 0.986 & 0.983 & 0.973 & 0.97 \\
\hline KNN & 0.934 & 0.864 & 0.788 & 0.817 \\
\hline
\end{tabular}

420 Table 4. Median F1 of the eight scRNAseq data classifier and the four conventional classifiers tools

421 on the four experiments $(N=\mathbf{1 0 0 0})$

\begin{tabular}{|l|l|l|l|l|}
\hline Training Dataset & $\begin{array}{l}\text { BaronHuman+ } \\
\text { Muraro+ Segerstolpe }\end{array}$ & $\begin{array}{l}\text { Xin+ Muraro+ } \\
\text { Segerstolpe }\end{array}$ & $\begin{array}{l}\text { Xin+ BaronHuman+ } \\
\text { Segerstolpe }\end{array}$ & $\begin{array}{l}\text { Xin+ } \\
\text { BaronHuman+ } \\
\text { Muraro }\end{array}$ \\
\hline Testing Dataset & Xin & BaronHuman & Muraro & Segerstolpe \\
\hline sigGCN & $\mathbf{0 . 9 9 3}$ & 0.976 & 0.957 & 0.989 \\
\hline scID & 0.991 & $\mathbf{0 . 9 8}$ & $\mathbf{0 . 9 7 6}$ & $\mathbf{0 . 9 9 4}$ \\
\hline
\end{tabular}




\begin{tabular}{|l|l|l|l|l|}
\hline scPred & 0.923 & 0.231 & 0.912 & 0.725 \\
\hline CasTLe & 0.974 & 0.916 & 0.966 & 0.988 \\
\hline SingleR & 0.988 & 0.974 & 0.969 & 0.994 \\
\hline scmapcluster & 0.133 & 0.001 & 0.001 & 0 \\
\hline scmapcell & 0.415 & 0.232 & 0.353 & 0.241 \\
\hline ACTINN & 0.982 & 0.977 & 0.957 & 0.989 \\
\hline RF & 0.946 & 0.914 & 0.957 & 0.903 \\
\hline SVM-linear & 0.986 & 0.972 & 0.952 & 0.986 \\
\hline SVM-rbf & 0.963 & 0.97 & 0.958 & 0.952 \\
\hline KNN & 0.766 & 0.74 & 0.504 & 0.602 \\
\hline
\end{tabular}

\section{Performance of runtimes}

424 We compared the runtimes of our method and the other scRNAseq classifiers. The runtimes were 425 computed by using an iMac with a $4.2 \mathrm{GHz}$ Intel Core i7 CPU and up to 32 gigabytes of memory. The 426 average runtime of each method using the Zhengsorted dataset is shown in Figure 6. The runtimes of all 427 the methods using the other six datasets are shown in Additional file 1: Figure S15. The average runtimes 428 of sigGCN are 404.3s, 1237.8s, 164.0s, 41.9s, 31.3s, 35.5s, 19.7s using Zhengsorted, Zheng68K, Baron 429 Human, Muraro, Segerstolpe, Baron Mouse, and Xin datasets, respectively. For the five pancreas dataset 430 that have a small number of cells, all the methods run less than 10 mins. For the large scale datasets, 431 Zhengsorted with 20,000 cells and Zheng68K with more than 60,000 cells, the average runtimes of our 432 method are less than or comparable to those of scID, CasTLe, singleR, and ACTINN. The runtimes of 433 scmapcluster and scmapcell are always low, even on the Zheng68K dataset, because scmapcluster assigns 434 the labels only by computing the distance between the testing cells and a small group of virtual cells.

\section{Evaluation of sensitivity to the number of genes}

437 We evaluated the sensitivity of the methods to the number of input genes in this section. We used the 438 same subset of genes which was selected based on the gene expression variance across the Zhengsorted 439 dataset as the input of all the scRNAseq classifiers including sigGCN. We selected top 300, 500, 750, $4401000,1250,1500$, and 5000 highest variant genes. The accuracy and median F1 of using a different

441 number of genes using the Zhengsorted dataset are shown in Figure 7. As can be seen, our method, 442 sigGCN, has a consistent performance in terms of accuracy and median F1 when using different sizes of 
443 the input features (high variant genes). The model performance for using a large size of genes $(>5,000)$

444 does not show a significant improvement compared with that of using a small size of genes $(\leq 1,000)$.

445 Considering the time complexity, we selected the top 1,000 variable genes as the default input in our

446 method.

\section{Evaluation of scalability to the number of cells}

449 To investigate the model scalability to different size of the dataset, we down-sampled the Zhengsorted

450 dataset into five subsets with the sampling rate of $0.05,0.1,0.15,0.25,0.5$. The ten cell classes of the

451 Zhengsorted dataset were down-sampled in a stratified way to guarantee that each cell class has the same

452 percentage. Therefore, the five subsets of Zhengsorted dataset have 1,000 cells, 2,000 cells, 3,000 cells,

4535,000 cells, and 10,000 cells. We divided each down-sampled subset to $80 \%$ as the training dataset, $10 \%$

454 as the validation dataset, and $10 \%$ as the testing dataset. The performance in terms of accuracy and

455 median F1 of the scRNAseq classifiers is shown in Figure 8. In general, the accuracy and median F1

456 scores of all the methods increase as the number of cells increases. Our method can hold consistent

457 median F1 on all the down-sampled subsets.

\section{Discussion and Conclusion}

460 In this study, we propose a novel method based on the deep learning methodologies for cell classification

461 in single cell studies when a large number of cells are used and data are heterogeneous with many classes.

462 We integrated the prior knowledge, in the form of gene-gene interaction network, into the classification

463 procedure. We developed an end-to-end trained multimodal deep learning model which includes a GCN

464 and an NN. The GCN model exploits the gene network and gene expression values to extract the 465 integrated features regarding the interconnection between genes, while the NN model is utilized to enrich 
467 We compared the performance of our proposed method with those of seven other classification

468 methods and four traditional machine learning classification methods using seven real single cell RNAseq

469 datasets. The standard classification metrics, including accuracy, median F1, median precision, and

470 median recall scores were utilized to evaluate the performance of the methods on all the 7 datasets. We

471 further evaluated the classification performance on a large and highly heterogeneous dataset, the

472 Zhengsorted dataset, by comparing the confusion matrix and the ROC plot. Results show that our

473 proposed method, sigGCN, outperforms both the current scRNAseq classification tools and the

474 conventional machine learning methods.

475 The main contribution of this work is integrating the prior knowledge in the form of the interaction

476 between genes into the classification of cell types. By aggregating the features from neighboring nodes in

477 the graph, the new features are learned in a nonlinear manner. The feature representation of the data using

478 a GCN is not redundant with that learned by using only an NN due to convolution through the gene

479 interaction structure. Therefore, we proposed to utilize a parallel structure of a GCN and an NN to

480 integrate the gene interaction structure and gene expression values.

481 In conclusion, the proposed multimodal deep learning model which integrates gene expression data 482 with the biological network to classifying cells shows a powerful performance. In the future work, we will 483 introduce the attention mechanism to enhance the weights of important genes.

\section{$487 \quad$ List of abbreviations}

488 scRNAseq: Single Cell RNA Sequencing

489 GCN: Graph convolutional network

490 NN: Neural network

491 ROC: Receiver Operating Characteristic 
492 TP: True Positive

493 FP: False Positive

494 TN: True Negative

495 FN: False Negative

496 AUC: Area Under Curve

497

498 Declarations

499 Ethics approval and consent to participate

500 No ethics approval was required for the study.

501

502 Consent for publication

503 Not applicable.

504

505 Availability of data and materials

506 The single cell RNAseq data are downloaded from https://doi.org/10.5281/zenodo.3357167 [25]. The

507 code is available at https://github.com/NabaviLab/sigGCN

508

\section{Competing Interests}

510 The authors declare that they have no competing interests.

\section{$512 \quad$ Funding}

513 This study was supported by the National Institutes of Health (NIH) grant No. R00LM011595, PI: Nabavi

514 and the National Science Foundation (NSF) under grant No. 1942303, PI: Nabavi.

\section{5}

\section{Authors' contributions}


517 SN and TW designed the study. TW and JB designed the models. TW collected the data and implemented

518 the analysis. TW, SN, and JB wrote the manuscript. All authors read and confirmed the manuscript.

520 Acknowledgments

521 Not applicable.

\section{Additional Files}

524 Additional file 1: Supplementary materials (Supplementary Tables S1-S4, Supplementary Figures S1-

525 S15).

526 Additional file 2: Additional_file_2_results_within-dataset_excluding_unassigned_cells, results of the

527 classification accuracy, F1 scores, and unassigned rate of not including the unassigned cells for five

528 methods (scPred, scID, scmapcluster, scmapcell, and sigGCN) using within-dataset experiments.

529 Additional file 3: Additional_file_2_results_cross-dataset_excluding_unassigned_cells, results of the

530 classification accuracy, F1 scores, and unassigned rate of not including the unassigned cells for five

531 methods (scPred, scID, scmapcluster, scmapcell, and sigGCN) using cross-dataset experiments.

\section{References}

534 1. Villani A-C, Satija R, Reynolds G, Sarkizova S, Shekhar K, Fletcher J, et al. Single-cell RNA-seq reveals new 535 types of human blood dendritic cells, monocytes, and progenitors. Science. 2017;356. doi:10.1126/science.aah4573.

536 2. Grün D, Lyubimova A, Kester L, Wiebrands K, Basak O, Sasaki N, et al. Single-cell messenger RNA sequencing 537 reveals rare intestinal cell types. Nature. 2015;525:251-5.

538 3. Segerstolpe Å, Palasantza A, Eliasson P, Andersson E-M, Andréasson A-C, Sun X, et al. Single-Cell

539 Transcriptome Profiling of Human Pancreatic Islets in Health and Type 2 Diabetes. Cell Metab. 2016;24:593-607.

540 4. Fincher CT, Wurtzel O, Hoog T de, Kravarik KM, Reddien PW. Cell type transcriptome atlas for the planarian 541 Schmidtea mediterranea. Science. 2018;360. doi:10.1126/science.aaq1736.

542 5. Plass M, Solana J, Wolf FA, Ayoub S, Misios A, Glažar P, et al. Cell type atlas and lineage tree of a whole 543 complex animal by single-cell transcriptomics. Science. 2018;360. doi:10.1126/science.aaq1723.

544 6. Zhao X, Wu S, Fang N, Sun X, Fan J. Evaluation of single-cell classifiers for single-cell RNA sequencing data 545 sets. Brief Bioinform. 2019;:bbz096. 
7. Alquicira-Hernandez J, Sathe A, Ji HP, Nguyen Q, Powell JE. scPred: accurate supervised method for cell-type classification from single-cell RNA-seq data. Genome Biol. 2019;20:264.

548 8. Boufea K, Seth S, Batada NN. scID: Identification of equivalent transcriptional cell populations across single cell RNA-seq data using discriminant analysis. bioRxiv. 2019;:470203.

9. Lieberman Y, Rokach L, Shay T. CaSTLe - Classification of single cells by transfer learning: Harnessing the power of publicly available single cell RNA sequencing experiments to annotate new experiments. PLOS ONE. 2018;13:e0205499.

553 10. Tan Y, Cahan P. SingleCellNet: a computational tool to classify single cell RNA-Seq data across platforms and 554 across species. preprint. Bioinformatics; 2018. doi:10.1101/508085.

11. Chen T, Guestrin C. XGBoost: A Scalable Tree Boosting System. Proc 22nd ACM SIGKDD Int Conf Knowl

556 Discov Data Min. 2016;:785-94.

557 12. Aran D, Looney AP, Liu L, Wu E, Fong V, Hsu A, et al. Reference-based analysis of lung single-cell

558 sequencing reveals a transitional profibrotic macrophage. Nat Immunol. 2019;20:163-72.

559 13. Kiselev VY, Yiu A, Hemberg M. scmap: projection of single-cell RNA-seq data across data sets. Nat Methods. $560 \quad 2018 ; 15: 359-62$.

14. Wagner F, Yanai I. Moana: A robust and scalable cell type classification framework for single-cell RNA-Seq data. preprint. Bioinformatics; 2018. doi:10.1101/456129.

563 15. Zhang Z, Danni Luo MS, Zhong X, Choi JH, Ma Y, Mahrt E, et al. SCINA: Semi-Supervised Analysis of Single 564 Cells in silico. preprint. Bioinformatics; 2019. doi:10.1101/559872.

565 16. Ma F, Pellegrini M. ACTINN: automated identification of cell types in single cell RNA sequencing. Bioinforma 566 Oxf Engl. 2020;36:533-8.

567 17. Wu Z, Pan S, Chen F, Long G, Zhang C, Yu PS. A Comprehensive Survey on Graph Neural Networks. IEEE

568 Trans Neural Netw Learn Syst. 2020;:1-21.

569 18. Defferrard M, Bresson X, Vandergheynst P. Convolutional neural networks on graphs with fast localized spectral filtering. In: Proceedings of the 30th International Conference on Neural Information Processing Systems. Barcelona, Spain: Curran Associates Inc.; 2016. p. 3844-52.

19. Fout A, Byrd J, Shariat B, Ben-Hur A. Protein Interface Prediction using Graph Convolutional Networks. In: Guyon I, Luxburg UV, Bengio S, Wallach H, Fergus R, Vishwanathan S, et al., editors. Advances in Neural 575 protein-interface-prediction-using-graph-convolutional-networks.pdf. Accessed 1 Jun 2020.

576 20. Sun M, Zhao S, Gilvary C, Elemento O, Zhou J, Wang F. Graph convolutional networks for computational drug 577 development and discovery. Brief Bioinform. 2020;21:919-35.

21. Szklarczyk D, Gable AL, Lyon D, Junge A, Wyder S, Huerta-Cepas J, et al. STRING v11: protein-protein association networks with increased coverage, supporting functional discovery in genome-wide experimental datasets. Nucleic Acids Res. 2019;47:D607-13.

581 22. Shuman DI, Narang SK, Frossard P, Ortega A, Vandergheynst P. The Emerging Field of Signal Processing on 582 Graphs: Extending High-Dimensional Data Analysis to Networks and Other Irregular Domains. IEEE Signal

583 Process Mag. 2013;30:83-98. 
584 23. Hammond DK, Vandergheynst P, Gribonval R. Wavelets on Graphs via Spectral Graph Theory. ArXiv09123848

585 Cs Math. 2009. http://arxiv.org/abs/0912.3848. Accessed 18 May 2020.

586 24. Ruder S. An overview of gradient descent optimization algorithms. ArXiv160904747 Cs. 2017.

587 http://arxiv.org/abs/1609.04747. Accessed 5 Dec 2020.

588 25. Abdelaal T, Michielsen L, Cats D, Hoogduin D, Mei H, Reinders MJT, et al. A comparison of automatic cell

589 identification methods for single-cell RNA sequencing data. Genome Biol. 2019;20:194.

590 26. Baron M, Veres A, Wolock SL, Faust AL, Gaujoux R, Vetere A, et al. A Single-Cell Transcriptomic Map of the

591 Human and Mouse Pancreas Reveals Inter- and Intra-cell Population Structure. Cell Syst. 2016;3:346-360.e4.

592 27. Muraro MJ, Dharmadhikari G, Grün D, Groen N, Dielen T, Jansen E, et al. A Single-Cell Transcriptome Atlas

593 of the Human Pancreas. Cell Syst. 2016;3:385-394.e3.

594 28. Xin Y, Kim J, Okamoto H, Ni M, Wei Y, Adler C, et al. RNA Sequencing of Single Human Islet Cells Reveals

595 Type 2 Diabetes Genes. Cell Metab. 2016;24:608-15.

596 29. Zheng GXY, Terry JM, Belgrader P, Ryvkin P, Bent ZW, Wilson R, et al. Massively parallel digital

597 transcriptional profiling of single cells. Nat Commun. 2017;8:14049.

598 30. 10x Genomics: Resolving Biology to Advance Human Health. https://www.10xgenomics.com/. Accessed 10 Jan 5992020.

600 31. Pedregosa F, Varoquaux G, Gramfort A, Michel V, Thirion B, Grisel O, et al. Scikit-learn: Machine Learning in 601 Python. Mach Learn PYTHON. :6.

602 32. Hanley JA, McNeil BJ. The meaning and use of the area under a Receiver Operating Characteristic (ROC) curve.

603 Radiology. 1982;143:29-36. 
617 Figures

618

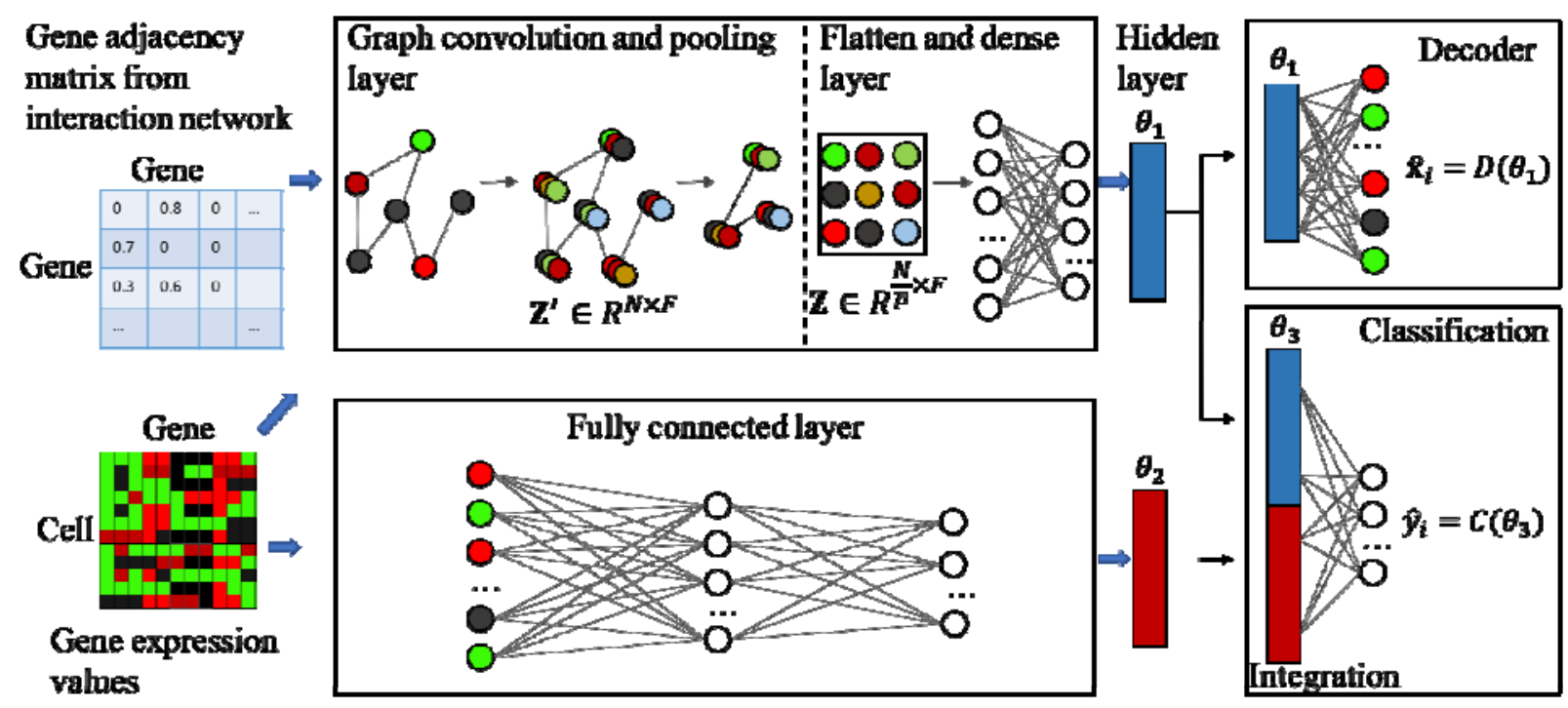

619 Figure 1: Structure of the proposed deep learning network (sigGCN) for single cell classification.

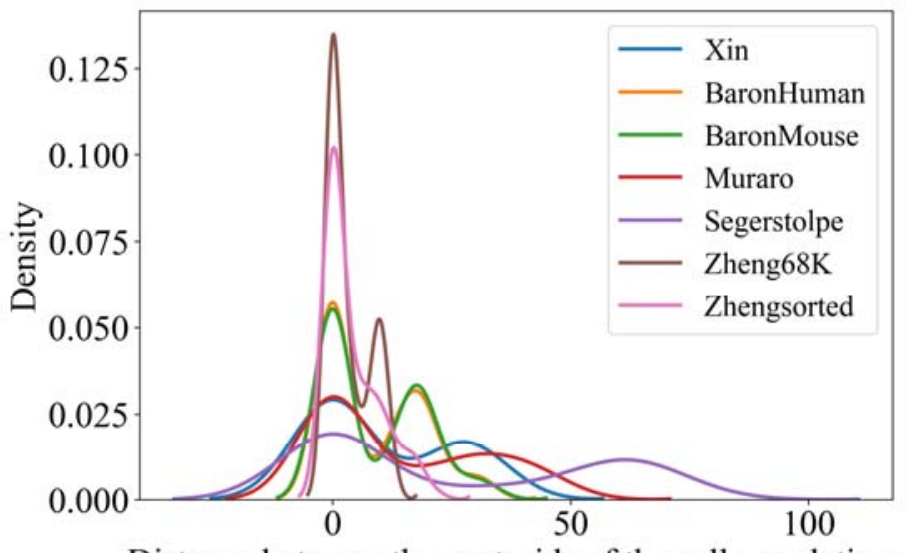

Distance between the centroids of the cell populations

621 Figure 2: Density plot of the distance between the centroids of cell populations to show the complexity of 622 the datasets. 


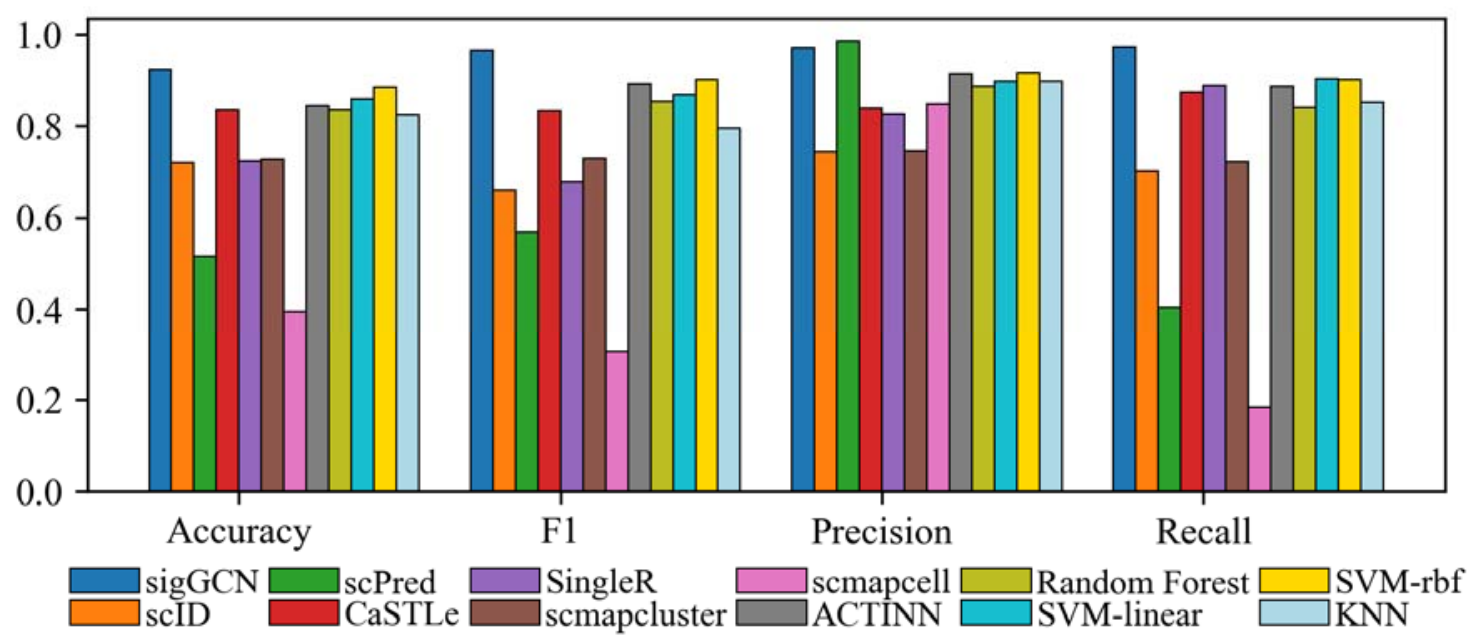

625 Figure 3: Bar plots of the four metrics to show the performance of the scRNAseq data classifier tools and conventional classifiers on Zhengsorted dataset.

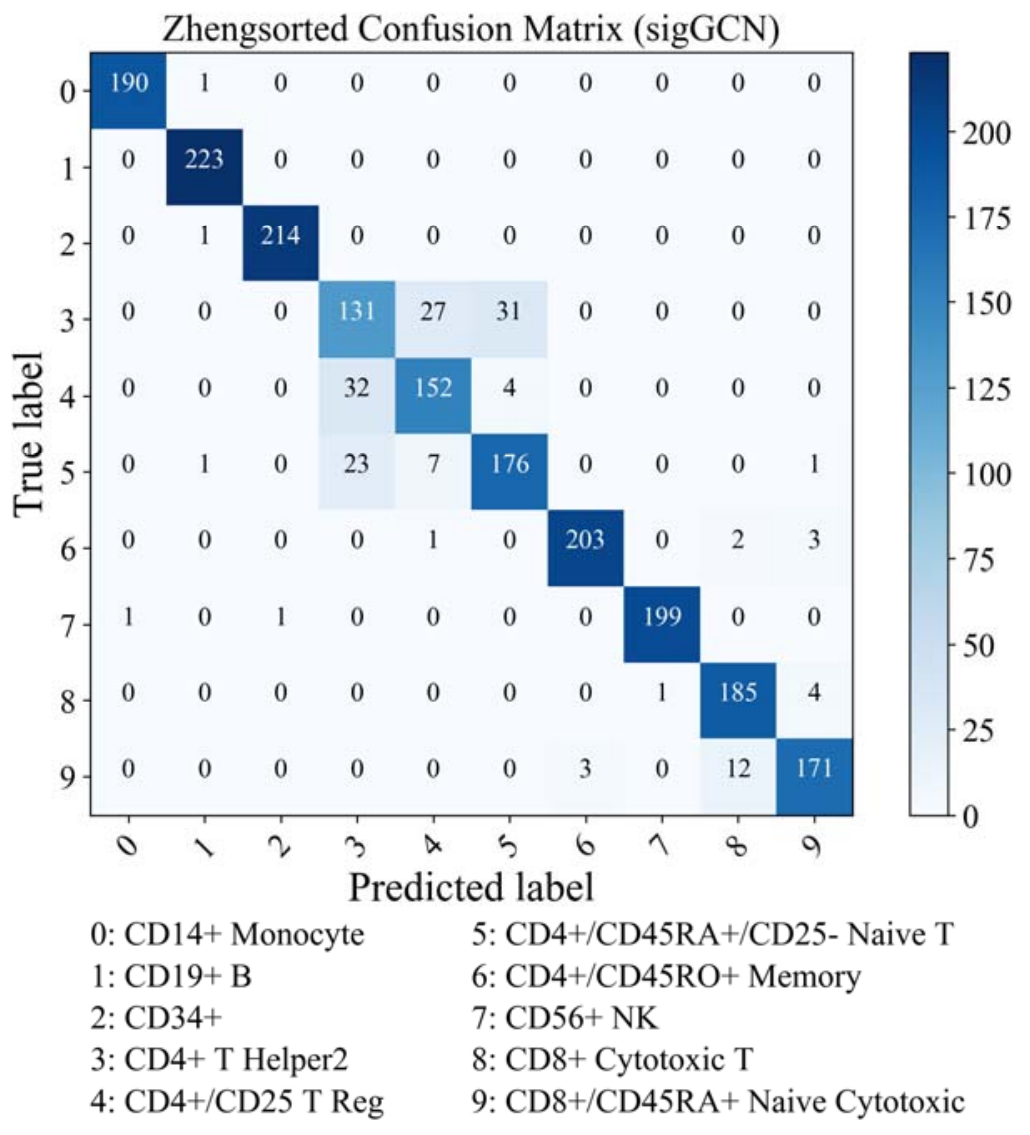

629 Figure 4: Confusion matrix of the class predictions on the Zhengsorted dataset using sigGCN. 

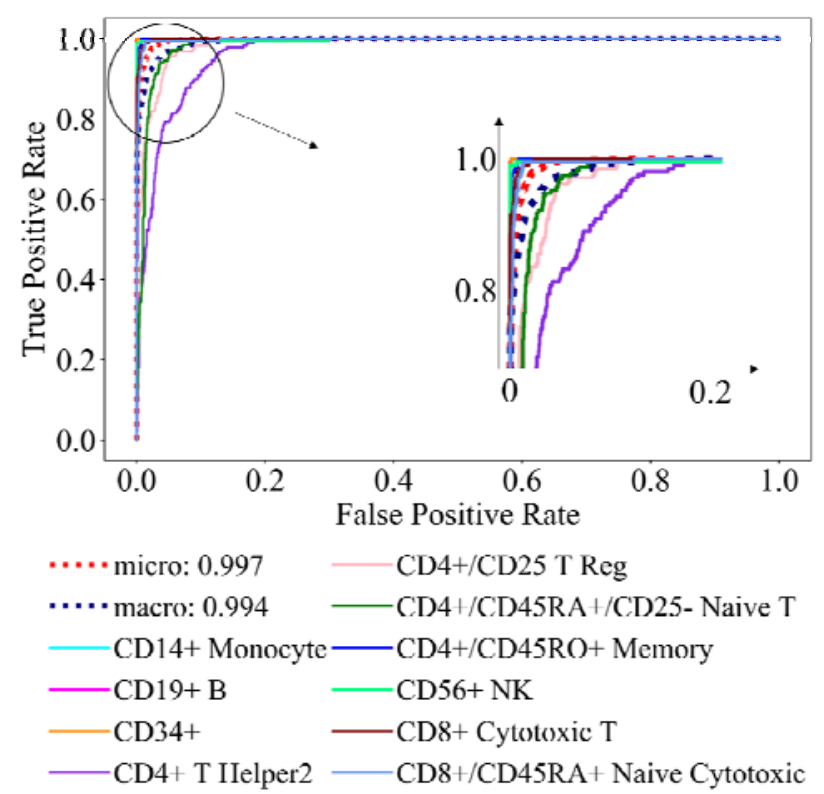

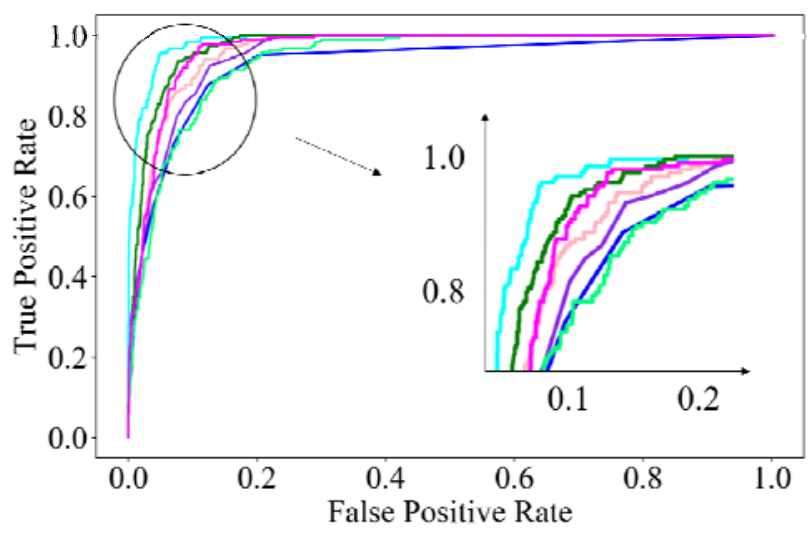

$\operatorname{sigGCN}(\mathrm{AUC}=0.9872)$

- RandomForest (AUC-0.9546, p-value-0.0072) SVM-linear $(\mathrm{AUC}=0.9618, \mathrm{p}$-value $=0.026)$ SVM-rbf ( $\triangle U \mathrm{UC}-0.9739$, p-value 0.1848 )

$-\mathrm{KNN}(\Lambda \mathrm{UC}=0.9288$, p-value $=0.0)$

CasTLc $(\mathrm{AUC}=0.9406$, p-valuc $=0.0005)$ $-\operatorname{scPred}(\Lambda \mathrm{UC}=0.9662, \mathrm{p}$-value $=0.0547)$

631 Figure 5: (a) Average AUC and ROC curves of sigGCN using the ten Zhengsorted cell types. (b) ROC curves of class 4 (CD4+/CD25 T Reg) and the p-values calculated by the McNeil \& Hanley's test that show the significance of difference between the areas under the ROC curve of sigGCN and that of each method.
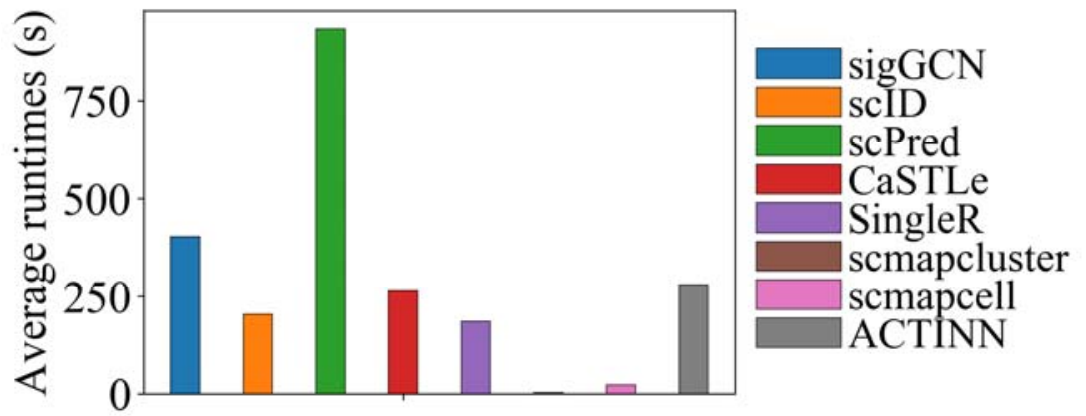

637 Figure 6: Average runtimes of the methods using Zhengsorted dataset. 
(a)

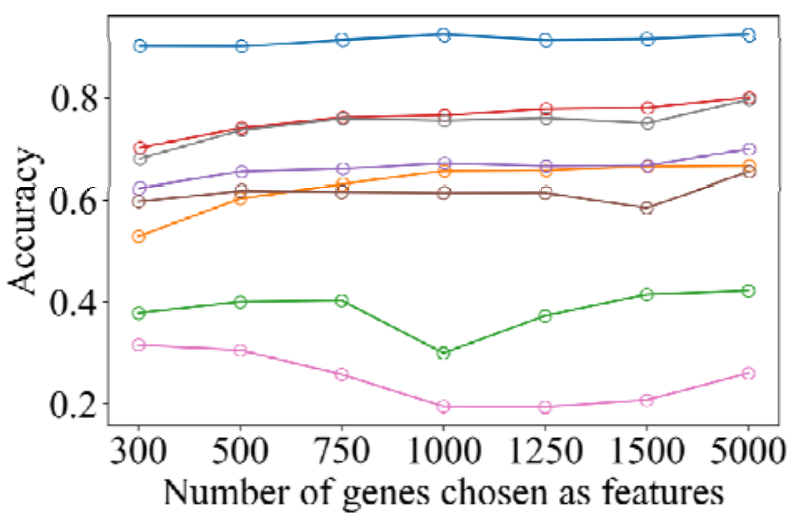

(b)

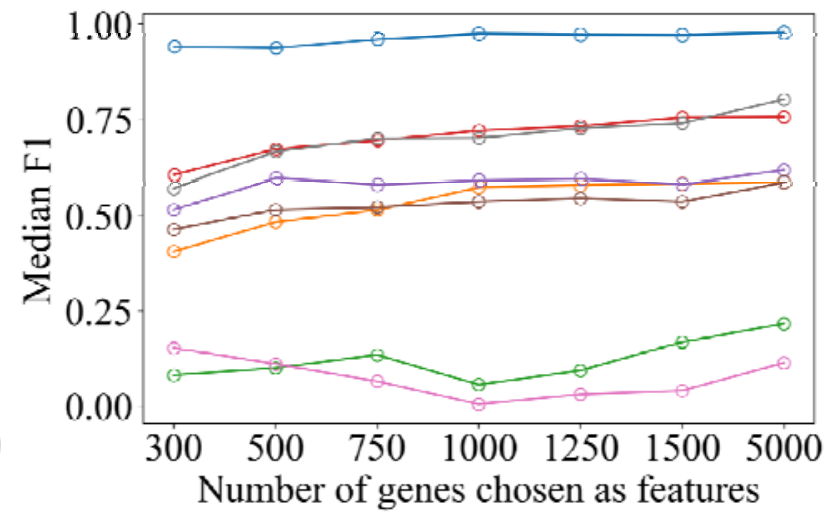

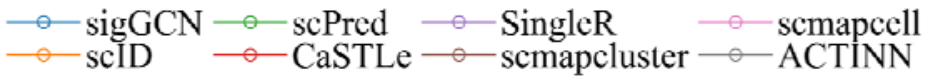

Figure 7: Results of choosing different numbers of genes as features of all the scRNAseq classifiers using 641

(a)

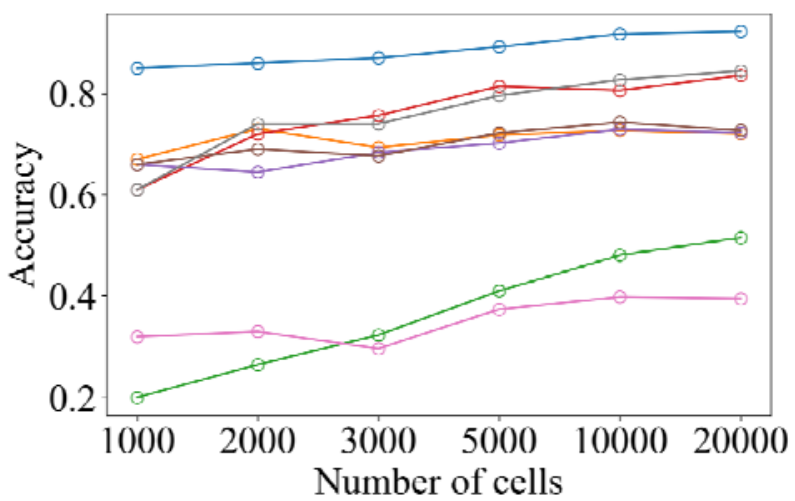

(b)

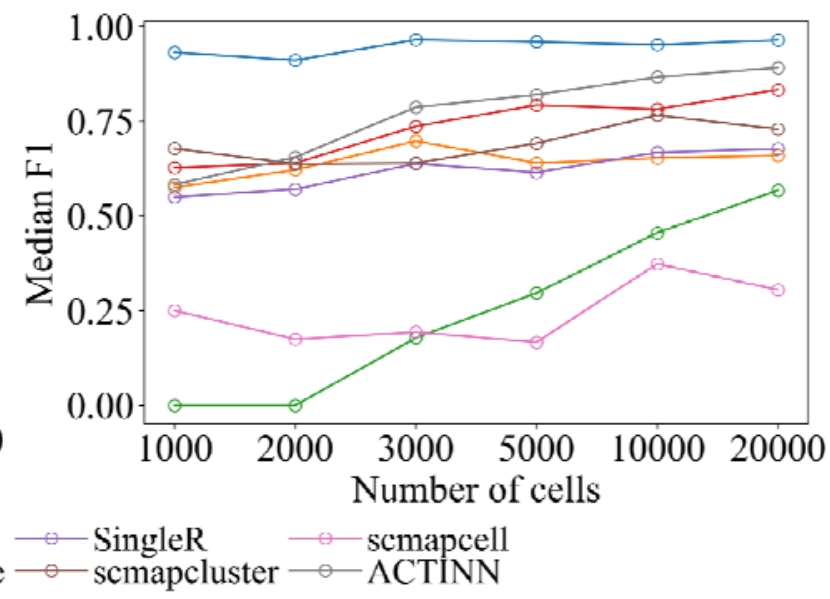

643 Figure 8: Results of all the scRNAseq classifiers using different numbers of cells from the Zhengsorted 644 dataset. 